\title{
The true quantum face of the "exponential" decay: Unstable systems in rest and in motion
}

\author{
K. Urbanowski ${ }^{1, a}$ \\ ${ }^{1}$ University of Zielona Góra, Institute of Physics, ul. Prof. Z. Szafrana 4a, 65-516 Zielona Góra, Poland.
}

\begin{abstract}
Results of theoretical studies and numerical calculations presented in the literature suggest that the survival probability $\mathcal{P}_{0}(t)$ has the exponential form starting from times much smaller than the lifetime $\tau$ up to times $t \gg \tau$, and that $\mathcal{P}_{0}(t)$ exhibits inverse power-law behavior at the late time region for times longer than the so-called crossover time $T \gg \tau$ (The crossover time $T$ is the time when the late time deviations of $\mathcal{P}_{0}(t)$ from the exponential form begin to dominate). More detailed analysis of the problem shows that in fact the survival probability $\mathcal{P}_{0}(t)$ can not take the pure exponential form at any time interval including times smaller than the lifetime $\tau$ or of the order of $\tau$ and it has has an oscillating form. We also study the survival probability of moving relativistic unstable particles with definite momentum $\vec{p} \neq 0$. These studies show that late time deviations of the survival probability of these particles from the exponential-like form of the decay law, that is the transition times region between exponential-like and non-exponential form of the survival probability, should occur much earlier than it follows from the classical standard considerations.
\end{abstract}

\section{Introduction}

The studies of the process of decay of radioactive elements were initialized by the discovery of radioactivity in the nineteenth century. Experiments have shown that the number of radioactive elements in the sample, which emits radioactive radiation, decreases with the passage of time. These observations and assumption that the decay rate follows the laws of probability led Rutherford and Sody to the formulation of radioactive decay law as a function of time [1-3]. This radioactive decay law allows to determine the number $N(t)$ of atoms of the radioactive element at the instant $t$ knowing the initial number $N_{0}=N(0)$ of them at initial instant of time $t_{0}=0$ and has the exponential form: $N(t)=N_{0} \exp [-\lambda t]$, where $\lambda>0$ is a constant. Since then, the belief that the decay law has the exponential form has become common. The rise of Quantum Mechanics led to an understanding that the radioactive decay similarly to the process of emission of photons by excited atoms are time dependent quantum processes. Considering the excited atomic levels and applying the Shrödinger equation to describe the time evolution Weisskopf and Wigner found that to a good approximation the non-decay probability of the exited levels is a decreasing function of time having exponential form [4]. Further studies of the quantum decay process showed that basic principles of the quantum theory does not allow it to be described by an exponential decay law at very late times $[5,6]$ and at

\footnotetext{
ae-mail: K.Urbanowski@if.uz.zgora.pl;k.a.urbanowski@gmail.com
} 
initial stage of the decay process (see [6] and references therein). Theoretical analysis shows that at late times the survival probability (i. e. the decay law) should tends to zero as $t \rightarrow \infty$ much more slowly than any exponential function of time and that as function of time it has the inverse power-like form at this regime of time $[5,6]$. There was many unsuccessful attempts to verify experimentally predicted deviations from the exponential form of the decay law at late times regime (see eg. [7]). The first experimental evidence of deviations of the decay law from exponential form at long time regime was reported in [8]. The early times properties of the decay process lead to the so called Quantum Zeno Effect $[9,10]$, that is to slowing down sufficiently frequently observed decay process up to stop it down in the case of the continuously observed an unstable system. The experimental confirmation of this effect was reported, e.g. in [11] and recently in [12]. All these results of theoretical and experimental researches caused that there are rather widespread belief that a universal feature of the quantum decay process is the presence of three time regimes of the decay process: the early time (initial), exponential (or "canonical"), and late time having inverse-power law form [13]. This belief is reinforced by a numerous presentations in the literature decay curves obtained for quantum models of unstable systems. The typical form of such a decay curve one can find in Fig. (1). In this context, each experimental evidence of oscillating decay curve at times of the order of life times is considered as an anomaly caused by a new quantum effects or new interactions: As an example of such a situation one can recall the result of the GSI experiment, where an oscillating decay rate of the ionized isotopes ${ }^{140} \mathrm{Pr}$ and ${ }^{142} \mathrm{Pm}$ moving with relativistic velocity $(\gamma \simeq 1.43$, where $\gamma$ is the Lorentz factor) was observed $[14,15]$. The question arises, if indeed in the case of one component quantum unstable systems these oscillations of the decay process at the "exponential" regime are an anomaly, or perhaps universal feature of quantum decay processes.

In the light of the mentioned results of the GSI experiment the another question arises: Whether and how the oscillations depend on the motion of the unstable quantum system. To find an answer to this question we need to know how to describe the decay process of unstable quantum systems in motion. This is quite general problem problem encountered by researchers studying properties of moving unstable particles: Namely physicists studying the decay processes are often confronted with the problem of how to predict the form of the decay law of the particle moving in respect to the rest reference frame of the observer knowing the decay law of this particle decaying in its rest frame. From the standard, text book considerations one finds that if the decay law of the unstable particle in rest has the exponential form $\mathcal{P}_{0}(t)=\exp \left[-\frac{\Gamma_{0} t}{\hbar}\right]$ then the decay law of the moving particle with momentum $p \neq 0$ is $\mathcal{P}_{p}(t)=\exp \left[-\frac{\Gamma_{0} t}{\hbar \gamma}\right]$, where $t$ denotes time, $\Gamma_{0}$ is the decay rate (time $t$ and $\Gamma_{0}$ are measured in the rest reference frame of the particle) and $\gamma$ is the relativistic Lorentz factor, $\gamma \equiv 1 / \sqrt{1-\beta^{2}}, \quad \beta=v / c, v=|\vec{v}|$ is the velocity of the particle, $\vec{v}=c \vec{p} / \sqrt{\vec{p}^{2}+m_{0}^{2} c^{2}}$ and $m_{0}-$ is the rest mass. This equality is the classical physics relation. It is almost common belief that this equality is valid also for any $t$ in the case of quantum decay processes and does not depend on the model of the unstable particles considered. For the proper interpretation of many accelerator experiments with high energy unstable particles as well as of results of observations of astrophysical processes in which a huge numbers of elementary particles (including unstable one) are produced we should be sure that this belief is supported by theoretical analysis of quantum models of decay processes. The problem seems to be extremely important because from some theoretical studies it follows that in the case of quantum decay processes this relation is valid to a sufficient accuracy only for not more than a few lifetimes $\tau_{0}=\hbar / \Gamma_{0}$ [16-19]. On the other hand all known tests of the relation $\mathcal{P}_{p}(t)=\exp \left[-\frac{\Gamma_{0} t}{\hbar \gamma}\right]$ were performed for times of the order of $\tau_{0}$ (see, eg. [20,21]) and for times longer than a few lifetimes this relation was not tested till now. What is more it appears that this relation may not apply in the case of the mentioned famous result of the GSI experiment. So we can see that the problem requires a deeper analysis. In these considerations the basis of such an analysis will be the formalism developed 
in $[16,17]$ where within the quantum field theory the formula for the survival amplitude of moving particles was derived. We will follow the method used in [19] and we will analyze numerically properties survival probability for a model of the unstable particle based on the Breit-Wigner mass distribution and considered therein.

So, in the following it will be shown that that in the case of unstable systems in rest there is no time interval in which the survival probability (decay law) could be a decreasing function of time of the purely exponential form. We also show that even in the case of a single component unstable system the decay curve has an oscillatory form with a smaller or a large amplitude of oscillations depending on the model considered. Next it will also be shown that the relativistic treatment of the problem within the Stefanovich-Shirokov theory $[16,17]$ yields decay curves tending to zero as $t \rightarrow \infty$ much slower than one would expect using classical time dilation relation which confirms and generalizes some conclusions drawn in [19]. Our results shows that conclusions relating to the quantum decay processes of moving particles based on the use of the classical physics time dilation relation need not be universally valid.

\section{Preliminaries}

The main information about properties of quantum unstable systems is contained in their survival probability. Let the reference frame $O_{0}$ be the common inertial rest frame for the observer and for the unstable system. Then if one knows that the system in the rest frame is in the initial unstable state $|\phi\rangle \in \mathcal{H},(\mathcal{H}$ is the Hilbert space of states of the considered system $)$, which was prepared at the initial instant $t_{0}=0$, one can calculate its survival probability (the decay law), $\mathcal{P}_{0}(t)$, of the unstable state $|\phi\rangle$ decaying in vacuum, which equals

$$
\mathcal{P}_{0}(t)=\left|a_{0}(t)\right|^{2},
$$

where $a_{0}(t)$ is the probability amplitude of finding the system at the time $t$ in the rest frame $O_{0}$ in the initial unstable state $|\phi\rangle$,

$$
a_{0}(t)=\langle\phi \mid \phi(t)\rangle
$$

and $|\phi(t)\rangle$ is the solution of the Schrödinger equation for the initial condition $|\phi(0)\rangle=|\phi\rangle$, which has the following form within the system units $\hbar=c=1$ used in the next parts of this paper:

$$
i \frac{\partial}{\partial t}|\phi(t)\rangle=H|\phi(t)\rangle
$$

where $|\phi\rangle,|\phi(t)\rangle \in \mathcal{H}$, and $H$ is the total self-adjoint Hamiltonian for the system considered. It is important to remember that if $|\phi\rangle$ represents an unstable state then it cannot be an eigenvector for $H$ : In such a case the eigenvalue equation $H|\phi\rangle=\epsilon_{\phi}|\phi\rangle$ has no solutions for $|\phi\rangle$ under considerations.

We have $|\phi(t)\rangle=U(t)|\phi\rangle$, where $U(t)$ is unitary evolution operator and $U(0)=\mathbb{I}$ is the unit operator. Therefore $a(t) \equiv\langle\phi|U(t)| \phi\rangle$. The one-parameter family of unitary operators $U(t)$ forms group: $U\left(t_{1}\right) U\left(t_{2}\right)=U\left(t_{1}+t_{2}\right)$. The the total Hamiltonian $H$ of the system is a generator of this group and operators $H$ and $U(t)$ have common eigenfunctions.

The rest reference frame $O_{0}$ is defined using common solution of the eigenvalue problem for $H$ and the momentum operator $\mathbf{P}$ :

$$
\mathbf{P}|\mu ; p\rangle=\vec{p}|\mu ; p\rangle
$$

and

$$
H|\mu ; p\rangle=E^{\prime}(\mu, p)|\mu ; p\rangle,
$$

where $\mu \equiv E^{\prime}(\mu, 0)$ and $\sigma_{c}(H)$ is the continuous part of the spectrum of the Hamiltonian $H$. Operators $H$ and $\mathbf{P}$ act in the state space $\mathcal{H}$. 
In the rest reference frame of the quantum unstable system $O_{0}$, when $\vec{p}=0$, we have $|\mu ; 0\rangle=$ $|\mu ; p=0\rangle$,

$$
\mathbf{P}|\mu ; 0\rangle=0,
$$

and

$$
H|\mu ; 0\rangle=\mu|\mu ; 0\rangle, \quad \mu \in \sigma_{c}(H),
$$

Eigenvectors $|\mu ; 0\rangle$ are normalized as usual:

$$
\left\langle 0 ; \mu \mid \mu^{\prime} ; 0\right\rangle=\delta\left(\mu-\mu^{\prime}\right) .
$$

Now we can model the unstable system in the rest system $\mathcal{O}_{0}$ as the following wave-packet $\left|\phi_{0}\right\rangle \equiv$ $\left|\phi_{\vec{p}=0}\right\rangle \stackrel{\text { def }}{=}|\phi\rangle$,

$$
\left|\phi_{0}\right\rangle \equiv|\phi\rangle=\int_{\mu_{0}}^{\infty} c(\mu)|\mu ; 0\rangle d \mu,
$$

where expansion coefficients $c(\mu)$ are functions of the mass parameter $\mu$, that is of the rest mass $\mu$. (Here $\mu_{0}$ is the lower bound of the spectrum $\sigma_{c}(H)$ of $H$ ). We require the state $\left|\phi_{0}\right\rangle$ to be normalized: So it has to be $\int_{\mu_{0}}^{\infty}|c(\mu)|^{2} d \mu=1$.

The expansion (9) and (7) allow one to find the amplitude $a_{0}(t)$ and to write

$$
a_{0}(t) \equiv \int_{\mu_{0}}^{\infty} \omega(\mu) e^{-i \mu t} d \mu,
$$

where $\omega(\mu) \equiv|c(\mu)|^{2}>0$.

So the amplitude $a_{0}(t)$, and thus the decay law $\mathcal{P}_{0}(t)$ of the unstable state $|\phi\rangle$, are completely determined by the density of the mass (energy) distribution $\omega(\mu)$ for the system in this state [22] (see also: [5, 6, 23-27]. From (10) and from the Riemann-Lebesque lemma it follows that $|a(t)| \rightarrow 0$ as $t \rightarrow \infty$. It is because from the normalization condition $a_{0}(0) \equiv \int_{S p e c .(H)} \omega(\mu) d m=1$ it follows that $\omega(\mu)$ is an absolutely integrable function. (Note that this approach is also applicable in Quantum Field Theory models $[28,29])$.

Khalfin assuming that the spectrum of $H$ must be bounded from below, $\mu_{0}>-\infty$, and using the Paley-Wiener Theorem [30] proved that in the case of unstable states there must be $\left|a_{0}(t)\right| \geq$ $A \exp \left[-b t^{q}\right]$, for $|t| \rightarrow \infty$, (see [5]). Here $A>0, b>0$ and $0<q<1$. Therefore the decay law $\mathcal{P}_{0}(t)$ of unstable states decaying in the vacuum, (1), can not be described by an exponential function of time $t$ if time $t$ is suitably long, $t \rightarrow \infty$, and that for these lengths of time $\mathcal{P}_{0}(t)$ tends to zero as $t \rightarrow \infty$ more slowly than any exponential function of $t$.

Note that the use of the Schrödinger equation (3) allows one to find that within the problem considered.

$$
i \frac{\partial}{\partial t}\langle\phi \mid \phi(t)\rangle=\langle\phi|H| \phi(t)\rangle .
$$

This relation leads to the conclusion that the amplitude $a_{0}(t)$ satisfies the following equation

$$
i \frac{\partial a_{0}(t)}{\partial t}=h(t) a_{0}(t)
$$

where

$$
h(t)=\frac{\langle\phi|H| \phi(t)\rangle}{a_{0}(t)},
$$

and $h(t)$ is the effective Hamiltonian governing the time evolution in the subspace of unstable states $\mathcal{H}_{\|}=P \mathcal{H}$, where $P=|\phi\rangle\langle\phi|$ (see [31] and also [32, 33] and references therein). The subspace 
$\mathcal{H} \ominus \mathcal{H}_{\|}=\mathcal{H}_{\perp} \equiv Q \mathcal{H}$ is the subspace of decay products. Here $Q=\mathbb{I}-P$. There is the following equivalent formula for $h(t)$ [31-33]:

$$
h(t) \equiv \frac{1}{a_{0}(t)} \frac{\partial a_{0}(t)}{\partial t}
$$

If $\langle\phi|H| \phi\rangle$ exists then using unitary evolution operator $U(t)$ and projection operators $P$ and $Q$ the relation (13) can be rewritten as follows

$$
h(t)=\langle\phi|H| \phi\rangle+\frac{\langle\phi|H Q U(t)| \phi\rangle}{a_{0}(t)} .
$$

One meets the effective Hamiltonian $h(t)$ when one starts with the Schrödinger equation for the total state space $\mathcal{H}$ and looks for the rigorous evolution equation for a distinguished subspace of states $\mathcal{H}_{\|} \subset \mathcal{H}[27,31]$. In general $h(t)$ is a complex function of time and in the case of $\mathcal{H}_{\|}$of dimension two or more the effective Hamiltonian governing the time evolution in such a subspace it is a nonhermitian matrix $H_{\|}$or non-hermitian operator. There is

$$
h(t)=\mu_{\phi}(t)-\frac{i}{2} \gamma_{\phi}(t)
$$

and

$$
\mu_{\phi}(t)=\mathfrak{R}[h(t)], \quad \gamma_{\phi}(t)=-2 \mathfrak{J}[h(t)],
$$

are the instantaneous mass (energy) $\mu_{\phi}(t)$ and the instantaneous decay rate, $\gamma_{\phi}(t)$ [31-33]. Here $\mathfrak{R}(z)$ and $\mathfrak{I}(z)$ denote the real and imaginary parts of $z$ respectively. The relations (12), (14) and (17) are convenient when the density $\omega(\mu)$ is given and one wants to find the instantaneous mass $\mu_{\phi}(t)$ and decay rate $\gamma_{\phi}(t)$ : Inserting $\omega(\mu)$ into (10) one obtains the amplitude $a_{0}(t)$ and then using (14) one finds the $h(t)$ and thus $\mu_{\phi}(t)$ and $\gamma_{\phi}(t)$.

In closing this Section we should pay attention to another problem: The state vector $|\phi\rangle$ of the form (9) corresponding to a quantum unstable system can not be an eigenvector of the Hamiltonian $H$, otherwise it would be that $\mathcal{P}_{0}(t)=|\langle\phi \mid \phi(t)\rangle|^{2}=|\langle\phi \mid \exp [-i t H] \phi\rangle|^{2} \equiv 1$ for all times $t$. The fact that the vector $|\phi\rangle$ describing the unstable quantum system is not the eigenvector for $H$ means that the mass (energy) of this object is not defined. Simply the mass can not take the exact constant value in this state $|\phi\rangle$. In such a case quantum systems are characterized by the mass (energy) distribution density $\omega(\mu)$ and the average mass $\left\langle m>=\int_{\mu_{0}}^{\infty} \mu \omega(\mu) d \mu\right.$ or by the instantaneous mass (energy) $\mu_{\phi}(t)$ but not by the exact value of the mass.

\section{The Breit-Wigner model}

It turns out that the decay curves obtained in the very large class of models are very similar in form to the curves calculated for $\omega(\mu)$ having a Breit-Wigner form (see [23] and analysis in [6]). So to find the most typical properties of the decay curve it is sufficient to make the relevant calculations for $\omega(\mu)$ modeled by the the Breit-Wigner distribution of the energy density $\omega_{B W}(\mu)$. The typical form of the survival probability $\mathcal{P}_{0}(t)$ obtained in such a way is presented in Fig (1), where the calculations were made for $\omega(\mu) \equiv \omega_{B W}(\mu)$,

$$
\omega_{B W}(\mu)=\frac{N}{2 \pi} \Theta\left(\mu-\mu_{0}\right) \frac{\Gamma_{0}}{\left(\mu-m_{0}\right)^{2}+\left(\frac{\Gamma_{0}}{2}\right)^{2}},
$$

where $N$ is a normalization constant. 


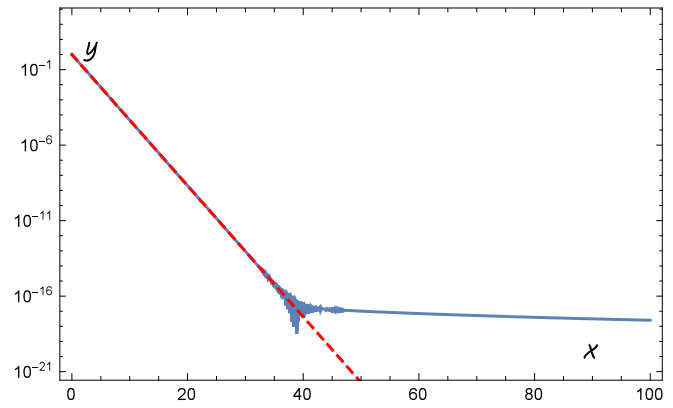

Figure 1. Decay curves obtained for $\omega_{B W}(E)$ given by Eq. (18). Axes: $x=t / \tau_{0}-$ time $t$ is measured in lifetimes $\tau_{0}, y$ - survival probabilities on a logarithmic scale (The solid line: the decay curve $\mathcal{P}_{0}(t)=\left|a_{0}(t)\right|^{2}$; The dotted line: the canonical decay curve $\mathcal{P}_{c}(t)=\left|a_{c}(t)\right|^{2}$. The case $s_{R}=\frac{E_{R}}{\Gamma_{0}}=1000$.

The standard canonical form of the survival amplitude $a_{c}(t)$, is given by the following relation,

$$
a_{c}(t)=\exp \left[-i \frac{t}{\hbar}\left(m_{0}-\frac{i}{2} \Gamma_{0}\right)\right]
$$

$\Gamma_{0}$ is the decay rate and $\frac{\hbar}{\Gamma_{0}} \equiv \frac{1}{\Gamma_{0}}=\tau_{0}$ is the lifetime within the assumed system of units $\hbar=c=1$ (time $t$ and $\Gamma_{0}$ are measured in the rest reference frame of the particle).

The case $\omega(\mu)=\omega_{B W}(\mu)$ is the typical case considered in numerous papers and used therein to model decay processes. Therefore it is very important to analyze real form of the decay curves obtained using $\omega(\mu)=\omega_{B W}(\mu)$ and this is why we consider this case in this paper. What is more inserting, substituting into $\omega_{B W}(\mu)$ into (10) allows one to find the analytical formula for the amplitude $a_{0}(t)$. The result is (see, eg. [32-34]),

$$
a_{0}(t)=N e^{-\frac{i}{\hbar}\left(m_{0}-i \frac{\Gamma_{0}}{2}\right) t}\left\{1-\frac{i}{2 \pi}\left[e^{\frac{\Gamma_{0} t}{\hbar}} E_{1}\left(-\frac{i}{\hbar}\left(m_{R}+\frac{i}{2} \Gamma_{0}\right) t\right)-E_{1}\left(-\frac{i}{\hbar}\left(m_{R}-\frac{i}{2} \Gamma_{0}\right) t\right)\right]\right\},
$$

where $E_{1}(x)$ denotes the integral-exponential function defined according to $[35,36]$ and $m_{R}=m_{0}-\mu_{0}$.

It is convenient to consider the following function

$$
\zeta(t) \stackrel{\text { def }}{=} \frac{a_{0}(t)}{a_{c}(t)} .
$$

There is

$$
|\zeta(t)|^{2}=\frac{\mathcal{P}_{0}(t)}{\mathcal{P}_{c}(t)}
$$

where $\mathcal{P}_{c}(t)=\left|a_{c}(t)\right|^{2}$ is the canonical exponential form of the decay law. Analysis of properties of this function allows one to visualize all the more subtle differences between $\mathcal{P}_{0}(t)$ and $\mathcal{P}_{c}(t)$. For example, if one finds a time interval $\left[t_{1}, t_{2}\right]$ such that $\zeta(t)=$ const for $t \in\left[t_{1}, t_{2}\right]$ this will mean that the survival probability $\mathcal{P}(t)$ has purely exponential form in this time interval.

The function $\zeta(t)$ takes the following form in the case of the unstable system modeled by $\omega_{B W}(m)$ :

$$
\zeta(t) \equiv N\left\{1-\frac{i}{2 \pi}\left[e^{\frac{\Gamma_{0} t}{\hbar}} E_{1}\left(-\frac{i}{\hbar}\left(m_{R}+\frac{i}{2} \Gamma_{0}\right) t\right)-E_{1}\left(-\frac{i}{\hbar}\left(m_{R}-\frac{i}{2} \Gamma_{0}\right) t\right)\right]\right\} .
$$


This function was used to find numerically $|\zeta(t)|^{2}$ for $\omega(m)=\omega_{B W}(m)$. Results of numerical calculations are presented in Figs (2) and (3): It turns out that in the case considered the form of $|\zeta(t)|^{2}$ and $\mathcal{P}_{0}(t)$ depend on the ratio $s_{R} \stackrel{\text { def }}{=} \frac{m_{R}}{\Gamma_{0}} \equiv \frac{m_{0}-\mu_{0}}{\Gamma_{0}}$.

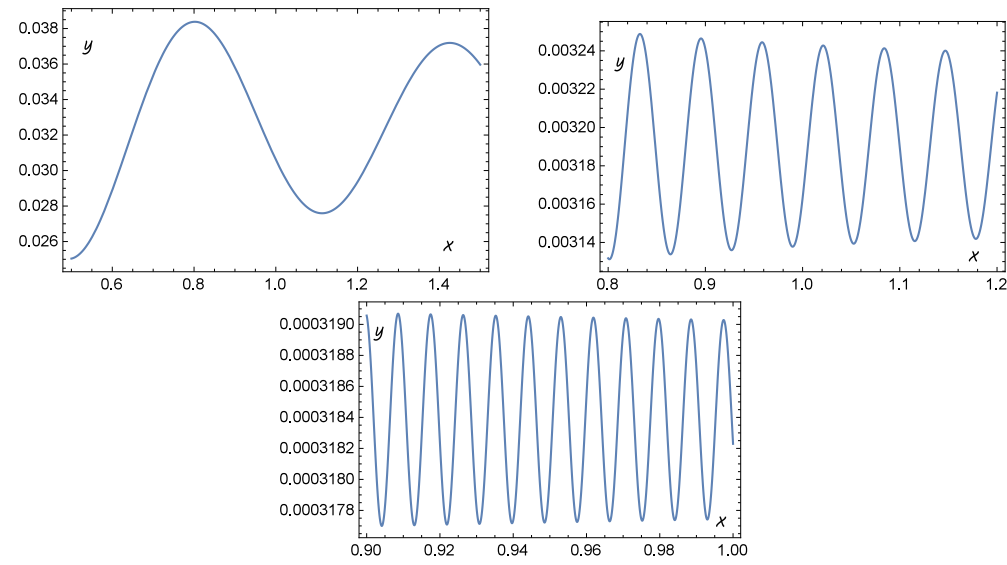

Figure 2. A comparison of decay curves obtained for $\omega_{B W}(\mu)$ given by Eq. (18) with canonical decay curves. Axes: $x=t / \tau_{0}$ - time $t$ is measured in lifetimes $\tau_{0}, y$ - The function $f(t)=\left(|\zeta(t)|^{2}-1\right)=\frac{\mathcal{P}_{0}(t)}{\mathcal{P}_{c}(t)}-1$, where $\zeta(t)$ is defined by the formula (21). The top panel: $s_{R}=10$. The middle panel: $s_{R}=100$. The lower panel: $s_{R}=1000$.

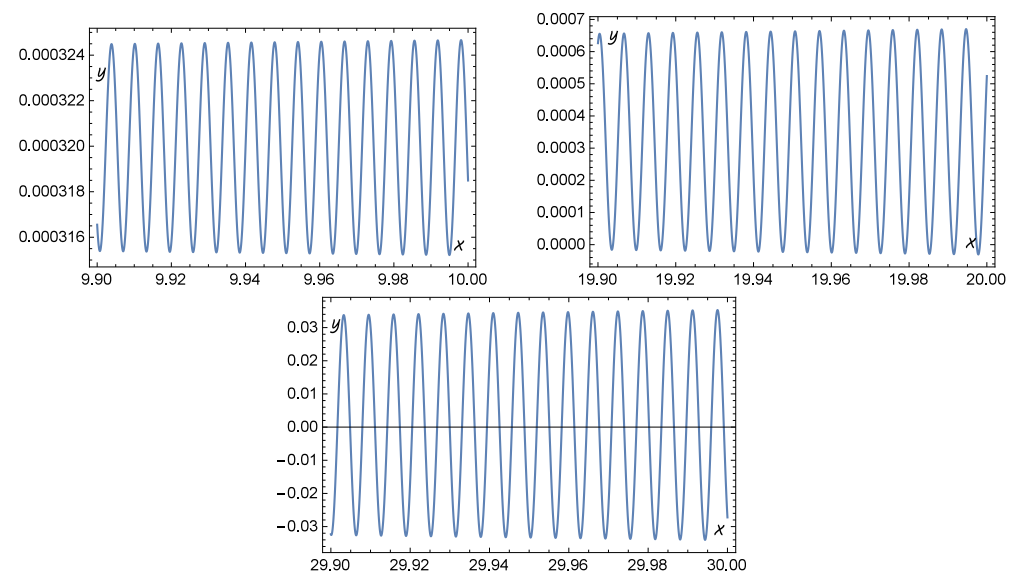

Figure 3. A comparison of decay curves obtained for $\omega_{B W}(\mu)$ given by Eq. (18) with canonical decay curves. Axes: $x=t / \tau_{0}$ - time $t$ is measured in lifetimes $\tau_{0}, y$ - The function $f(t)=\left(|\zeta(t)|^{2}-1\right)=\frac{\mathcal{P}_{0}(t)}{\mathcal{P}_{c}(t)}-1$, where $\zeta(t)$ is defined by the formula (21), $\mathcal{P}_{0}(t)=\left|a_{0}(t)\right|^{2}, \mathcal{P}_{c}(t)=\left|a_{c}(t)\right|^{2}$. The case $s_{R}=1000$.

The derivative of $\zeta(t)$ given by (23) equals $[32,33]$

$$
\frac{\partial \zeta(t)}{\partial t}=i \frac{N}{2 \pi} \frac{\Gamma_{0}}{\hbar} e^{\frac{\Gamma_{0}}{\hbar} t} E_{1}\left(-\frac{i}{\hbar}\left(m_{R}+\frac{i}{2} \Gamma_{0}\right) t\right) .
$$


From the properties of the integral-exponential function $E_{1}(x)$ it follows that the equation $\frac{\partial \zeta(t)}{\partial t}=0$ can be satisfied at most for some isolated values of time $t$. So, from the formula (24) the conclusion follows: Within the model considered there is no time interval in which $\zeta(t)=$ const, that is, there is no time interval in which the survival probability $\mathcal{P}_{0}(t)$ has a pure exponential form. This conclusion explains the results presented in Figs (2) and (3).

\section{The general case of $\zeta=\frac{a_{0}(t)}{a_{c}(t)}$}

Let us consider now the general case of $\zeta(t)$. From the definition (21) it follows that

$$
\frac{\partial \zeta(t)}{\partial t} \equiv \frac{i}{\hbar}\left(m_{0}-\frac{i}{2} \Gamma_{0}\right) \zeta(t)+e^{+i \frac{t}{\hbar}\left(m_{0}-\frac{i}{2} \Gamma_{0}\right)} \frac{\partial a(t)}{\partial t}=\frac{i}{\hbar}\left(m_{0}-\frac{i}{2} \Gamma_{0}\right) \zeta(t)-\frac{i}{\hbar} h(t) \zeta(t) .
$$

Let us use now the relation (15) and assume that $\langle\phi|H| \phi\rangle$ exists and there exists instants $0<t_{1}<$ $t_{2}<\infty$ of time $t$ such that for any $t \in\left(t_{1}, t_{2}\right)$ there is $\zeta(t)=\zeta\left(t_{1}\right)=\zeta\left(t_{2}\right)=$ const $\stackrel{\text { def }}{=} c_{\phi} \neq 0$. In this case there should be $\frac{\partial \zeta(t)}{\partial t}=0$ for all $t \in\left(t_{1}, t_{2}\right)$. Taking into account that by definition $\zeta(t) \neq 0$ from (25) we conclude that it is possible only and only if

$$
h(t)-\left(m_{0}-\frac{i}{2} \Gamma_{0}\right)=0, \text { for } t_{1} \leq t \leq t_{2}
$$

that is if and only if

$$
h\left(t_{1}\right)=h(t)=h\left(t_{2}\right)=\text { const } \stackrel{\text { def }}{=} c_{h} \neq 0 \text { for } t_{1} \leq t \leq t_{2} .
$$

Using (15) and the property $|\phi(t)\rangle=U(t)|\phi\rangle$ one concludes that the equality $h\left(t_{1}\right)=h(t)=c_{h}$ can take place if

$$
\frac{\left\langle\phi\left|H Q U\left(t_{1}\right)\right| \phi\right\rangle}{a_{0}\left(t_{1}\right)}=\frac{\langle\phi|H Q U(t)| \phi\rangle}{a_{0}(t)} .
$$

we can replace the operator $U(t)$ by the product $U\left(t_{1}\right) U\left(t-t_{1}\right) \equiv U(t)$. Taking into account the group properties of the one-parameter family of unitary operators $U(t)$ we can use in (28) $U\left(t_{1}\right) U\left(t-t_{1}\right) \equiv$ $U(t)$ instead of $U(t)$. Next keeping in mind that $a_{0}(t) \neq 0, a_{0}\left(t_{1}\right) \neq 0$ and taking into account that $\lambda\left(t, t_{1}\right) \stackrel{\text { def }}{=} \frac{a_{0}(t)}{a_{0}\left(t_{1}\right)}$ is a complex function one can replace the relation (28) by the following one

$$
\left\langle\phi \left| H Q U\left(t_{1}\right)\left[\lambda\left(t, t_{1}\right)|\phi\rangle-U\left(t-t_{1}\right)|\phi\rangle\right]=0 .\right.\right.
$$

This condition can be satisfied in two cases: The first one is

$$
U\left(t-t_{1}\right)|\phi\rangle-\lambda\left(t, t_{1}\right)|\phi\rangle=0,
$$

and the second occurs when $\left[\lambda\left(t, t_{1}\right)|\phi\rangle-U\left(t-t_{1}\right)|\phi\rangle\right] \neq 0$ and vectors $(\langle\phi| H)^{+}=H|\phi\rangle$ and $Q U\left(t_{1}\right)\left[\lambda\left(t, t_{1}\right)|\phi\rangle-U\left(t-t_{1}\right)|\phi\rangle\right]$ are orthogonal to each other.

The first case means that $h\left(t_{1}\right)=h(t)=c_{h}=$ const which by (27) means that $\frac{\partial \zeta(t)}{\partial t}=0$ if and only if the vector $|\phi\rangle$ representing an unstable state of the system is an eigenvector for the unitary evolution operator $U(t)$. As we noted earlier the evolution operator $U(t)$ and the total Hamiltonian $H$ of the system have common eigenvectors. This means that $h\left(t_{1}\right)=h(t)=c_{h}=$ const and thus $\frac{\partial \zeta(t)}{\partial t}=0$ for $t \in\left(t_{1}, t_{2}\right)$ if and only if the unstable state $|\phi\rangle$ of the system is an eigenvector for $H$, which is in contradiction with the property that the vector $|\phi\rangle$ representing the unstable state cannot be the eigenvector for the total Hamiltonian $H$. 
The second case: From the definition of the projectors $P$ and $Q$ it follows that this case can be realized only if the vector $H|\phi\rangle$ is proportional to the vector $|\phi\rangle: H|\phi\rangle=\alpha_{\phi}|\phi\rangle$, that is similarly to the first case $\frac{\partial \zeta(t)}{\partial t}=0$ if and only if the vector $|\phi\rangle$ representing the unstable state of the system considered is an eigenvector for the total Hamiltonian $H$, which is again in clear contradiction with the condition that the vector $|\phi\rangle$ representing the unstable state cannot be the eigenvector for the total Hamiltonian $H$.

Taking into account implications of the above to possible realizations of the relation (29) we conclude the supposition that such time interval $\left[t_{1}, t_{2}\right]$ can exist that $h\left(t_{1}\right)=h(t)=c_{h}=$ const for $t \in\left(t_{1}, t_{2}\right)$ and thus $\zeta(t)=$ const $=\zeta\left(t_{1}\right)=\zeta\left(t_{2}\right)$ for $t \in\left(t_{1}, t_{2}\right)$ is false. So taking into account the definition of $\zeta(t)$ the following conclusion follows: Within the approach considered in this paper for any time interval $\left[t_{1}, t_{2}\right]$ the decay law can not be described by the exponential function of time. This conclusion is the general one. It does not depend on models of quantum unstable states and confirms the similar conclusion drawn earlier for the Breit-Wigner model.

The another important conclusion is that at any time interval $\left[t_{1}, t_{2}\right]$ the effective Hamiltonian $h(t)$ can not be constant. This means that at any time interval $\left[t_{1}, t_{2}\right]$ the instantaneous mass $\mu_{\phi}(t)=\mathfrak{R}[h(t)]$ and decay rate $\gamma_{\phi}(t)=-2 \mathfrak{J}[h(t)]$ can not be constant in time:

$$
\mu_{\phi}(t) \neq \text { const. }, \quad \gamma_{\phi}(t) \neq \text { const } .
$$

In other words, as it follows from the above analysis the case $\mu_{\phi}(t)=$ const and $\gamma_{\phi}(t)=$ const can be realized only if the state $|\phi\rangle$ is an eigenvector for the total Hamiltonian $H$, that is if an only if there is no any decay od the state $|\phi\rangle$. Results of numerical calculations performed for $\omega(\mu)=\omega_{B W}(\mu)$ and presented in Fig (4) confirm the conclusion (31). In these Figures the function

$$
\kappa(t)=\frac{\mu_{\phi}(t)-\mu_{0}}{m_{0}-\mu_{0}},
$$

is presented and calculations were performed for $s_{R}=\frac{m_{R}}{\Gamma_{0}}=\frac{m_{0}-\mu_{0}}{\Gamma_{0}}=1000$. This function illustrates a typical form of time-varying $\mu_{\phi}(t)$.
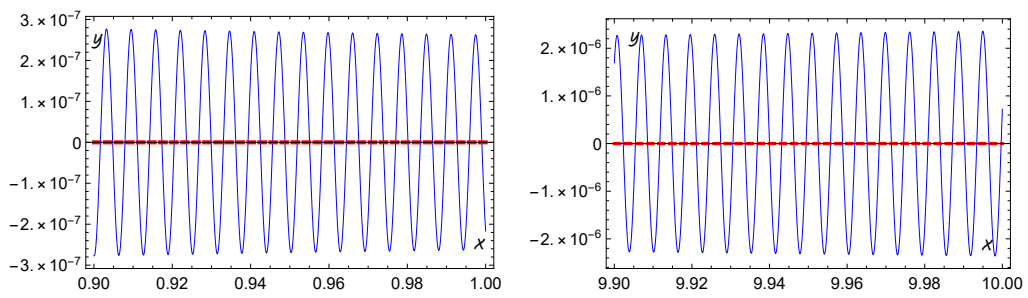

Figure 4. The instantaneous mass $m_{\phi}(t)$ as a function of time obtained for $\omega_{B W}(\mu)$. Axes: $y=\kappa(t)-1$, where $\kappa(t)$ is defined by (32); $x=t / \tau_{\phi}$ : Time is measured in lifetimes. The horizontal dashed line represents the value of $\mu_{\phi}(t)=m_{0}$

Summing up the oscillating decay curves of one component unstable system can not be considered as something extraordinary or as anomaly: It seems to be a universal feature of the decay process. The oscillatory modulation of decay curves takes place even in the quantum unstable system modeled by the Breit-Wigner distribution of the energy density. In general, the oscillatory modulation of the survival probability and thus the decay curves with model depending amplitude and oscillations 
period takes place even in the case of one component unstable systems. From results of the model calculations presented in Figs (2) and (3) it follows that at the initial stage of the "exponential" (or "canonical") decay regime the amplitude of these oscillations may be much less than the accuracy of detectors. Then with increasing time the amplitude of oscillations grows (see Fig. (3)), which increases the chances of observing them. This is a true quantum picture of the decay process at the so-called "exponential" regime of times.

\section{Moving unstable systems with constant momentum}

In the case of moving quantum unstable systems we need the probability amplitude $a_{p}(t)=\left\langle\phi_{p} \mid \phi_{p}(t)\right\rangle$, which defines the survival probability $\mathcal{P}_{p}(t)=\left|a_{p}(t)\right|^{2}$. There is $\left|\phi_{p}(t)\right\rangle \stackrel{\text { def }}{=} \exp [-i t H]\left|\phi_{p}\right\rangle$ in $\hbar=c=1$ units. So we need the vector $\left|\phi_{p}\right\rangle$ and eigenvalues $E^{\prime}(\mu, p)$ solving Eq. (5). Vectors $|\phi\rangle,\left|\phi_{p}\right\rangle$ are elements of the same state space $\mathcal{H}$ connected with the coordinate rest system of the observer $O$ : We are looking for the decay law of the moving particle measured by the observer $O$. If to assume for simplicity that $\mathbf{P}=\left(P_{1}, 0,0\right)$ and that $\vec{v}=\left(v_{1}, 0,0\right) \equiv(v, 0,0)$ then there is $\vec{p}=(p, 0,0)$ for the eigenvalues $\vec{p}$ of the momentum operator $\mathbf{P}$. Let $\Lambda_{p, \mu}$ be the Lorentz transformation from the reference frame $O$, where the momentum of the unstable particle considered is zero, $\vec{p}=0$, into the frame $O^{\prime}$ where the momentum of this particle is $\vec{p} \equiv(p, 0,0) \neq 0$ and $p \geq 0$, or, equivalently, where its velocity equals $\vec{v}=\vec{v}_{p, \mu} \equiv \frac{\vec{p}}{\mu \gamma_{\mu}}$, (where $\mu$ is the rest mass and $\gamma_{\mu} \equiv \sqrt{p^{2}+(\mu)^{2}} / \mu$ ). Now using the unitary representation, $U\left(\Lambda_{p, \mu}\right)$, of the transformation $\Lambda_{p, \mu}$, which acts in the Hilbert space $\mathcal{H}$ of states $|\phi\rangle \equiv|\phi ; 0\rangle,\left|\phi_{p}\right\rangle \in \mathcal{H}$, one can show that the vector $U\left(\Lambda_{p, \mu}\right)|\mu ; 0\rangle$ is the common eigenvector for operators $H$ and $\mathbf{P}$, that is that there is $|\mu ; p\rangle \equiv U\left(\Lambda_{p, \mu}\right)|\mu ; 0\rangle$ (see, eg. [37]). Indeed, taking into account that operators $H$ and $\mathbf{P}$ form a 4-vector $P_{v}=\left(P_{0}, \mathbf{P}\right) \equiv\left(P_{0}, P_{1}, 0,0\right)$, and $P_{0} \equiv H$, we have $U^{-1}\left(\Lambda_{p, \mu}\right) P_{v} U\left(\Lambda_{p, \mu}\right)=\Lambda_{p, \mu ; \nu} P_{\lambda}$, where $v, \lambda=0,1,2,3$ (see, e.g., [37], Chap. 4). From this general transformation rule it follows that

$$
U^{-1}\left(\Lambda_{p, \mu}\right) P_{0} U\left(\Lambda_{p, \mu}\right)=\gamma_{\mu}\left(P_{0}+v_{\mu} P_{1}\right) \equiv \gamma_{\mu}\left(H+v_{\mu} P_{1}\right)
$$

Based on the relation (33), one can show that that vectors $U\left(\Lambda_{p, \mu}\right)|\mu ; 0\rangle$ are eigenvectors for the Hamiltonian $H$. There is

$$
H U\left(\Lambda_{p, \mu}\right)|\mu ; 0\rangle=U\left(\Lambda_{p, \mu}\right) U^{-1}\left(\Lambda_{p, \mu}\right) H U\left(\Lambda_{p, \mu}\right)|\mu ; 0\rangle=\gamma_{\mu} U\left(\Lambda_{p, \mu}\right)\left(H+v_{\mu} P_{1}\right)|\mu ; 0\rangle .
$$

The Lorentz factor $\gamma_{\mu}$ corresponds to the rest mass $\mu$ being the eigenvalue of the vector $|\mu ; 0\rangle$. There are $\gamma_{\mu} \neq \gamma_{\mu^{\prime}}$ and $v_{\mu} \neq v_{\mu^{\prime}}$ for $\mu \neq \mu^{\prime}$. From (4), (6) it follows that $P_{1}|\mu ; 0\rangle=0$ for $p=0$, which means that using (7) the relation (34) can be rewritten as follows

$$
H U\left(\Lambda_{p, \mu}\right)|\mu ; 0\rangle=\mu \gamma_{\mu} U\left(\Lambda_{p, \mu}\right)|\mu ; 0\rangle
$$

Taking into account the form of the $\gamma_{\mu}$ forced by the condition $p=$ const one concludes that in fact the eigenvalue found, $\mu \gamma_{\mu}$, equals $\mu \gamma_{\mu} \equiv \sqrt{p^{2}+\mu^{2}}$. So, $E^{\prime}(\mu, p)=\sqrt{p^{2}+\mu^{2}}$. (Similarly one can show that vectors $U\left(\Lambda_{p, \mu}\right)|\mu ; 0\rangle$ are the eigenvectors of the momentum operator $\mathbf{P}$ for the eigenvalue $\mu \gamma_{\mu} v_{\mu} \equiv p$, that is that $\left.U\left(\Lambda_{p, \mu}\right)|\mu ; 0\rangle \equiv|\mu ; p\rangle\right)$. Thus finally we come to desired results:

$$
H|\mu ; p\rangle=\sqrt{p^{2}+\mu^{2}}|\mu ; p\rangle
$$

which replaces Eq. (5). 
The moving quantum unstable particle $\phi$ with constant momentum, $\vec{p}$, can be modeled analogously as the quantum unstable system in the rest frame (when $\vec{p}=0$ ) as the following wave-packet $\left|\phi_{p}\right\rangle$,

$$
\left|\phi_{p}\right\rangle=\int_{\mu_{0}}^{\infty} c(\mu)|\mu ; p\rangle d m
$$

where expansion coefficients $c(\mu)$ are functions of the mass parameter $\mu$, that is of the rest mass $\mu$, which is Lorentz invariant and therefore the scalar functions $c(\mu)$ of $\mu$ are also Lorentz invariant and are the same as in the rest reference frame $O_{0}$.

Now using (36) and the equation (37) we obtain the final, required relation for the amplitude $a_{p}(t)$ (see $[16,17,38])$,

$$
a_{p}(t)=\int_{\mu_{0}}^{\infty} \omega(\mu) e^{-i \mu \gamma_{\mu} t} d \mu \equiv \int_{\mu_{0}}^{\infty} \omega(\mu) e^{-i \sqrt{p^{2}+\mu^{2}} t} d \mu .
$$

This is the place when it should be explained why the Lorentz factor $\gamma_{\mu}$ is used in (38) (and earlier in relations (33) - (36) instead of $\gamma_{\mu}=\gamma$. In the rest reference frame the unstable quantum system is modeled as the wave packet given by the relation (9). Let us choose some eigenvalues, $\mu_{1}<\mu_{2}<$ $\ldots<\mu_{k}<\ldots<\mu_{n} \in \sigma_{c}(H)=\left[\mu_{0}, \infty\right)$, of the spectrum $\sigma_{c}(H)$ of $H$. These eigenvalues are connected with corresponding eigenvectors $\left|\mu_{k} ; 0\right\rangle$ of $H$ as follows:

$$
\begin{array}{ccccccc} 
& & O_{0} & \multicolumn{5}{c}{O_{p}} & & \\
\mu_{1} & \leftrightarrow & \left|\mu_{1} ; 0\right\rangle & \stackrel{U\left(\Lambda_{p ; \mu_{1}}\right)}{\longmapsto} & \left|\mu_{1} ; p\right\rangle & \leftrightarrow & p=\mu_{1} \gamma_{\mu_{1}} v_{\mu_{1}} \\
\mu_{2} & \leftrightarrow & \left|\mu_{2} ; 0\right\rangle & \stackrel{U\left(\Lambda_{p ; \mu_{2}}\right)}{\longmapsto} & \left|\mu_{2} ; p\right\rangle & \leftrightarrow & p=\mu_{2} \gamma_{\mu_{2}} v_{\mu_{2}} \\
\ldots & \ldots & \ldots & \ldots & \ldots & \ldots & \ldots \\
\mu_{k} & \leftrightarrow & \left|\mu_{k} ; 0\right\rangle & \stackrel{U\left(\Lambda_{p ; \mu_{k}}\right)}{\longmapsto} & \left|\mu_{k} ; p\right\rangle & \leftrightarrow & p=\mu_{k} \gamma_{\mu_{k}} v_{\mu_{k}} \\
\ldots & \ldots & \ldots & \ldots & \ldots & \ldots & \ldots
\end{array}
$$

As it is seen from the above analysis each vector $|\mu ; 0\rangle$ numbered by $\mu \in \sigma_{c}(H)$ can be transformed correctly in the vector $|\mu ; p\rangle$ connected with the reference frame $O_{p}$ only if one takes into account that every point $\mu$ from the spectrum of $H$, considered as the rest mass, has the "own" Lorentz factor $\gamma=\sqrt{\mu^{2}+p^{2}} / \mu \equiv \gamma_{\mu}$. (The reference frame $O_{p}$ was defined by condition $\vec{p}=$ const $\neq 0$, and $O_{0}=O_{p=0}$ ). In other words it is impossible to realize the above transformations of vectors $|\mu ; 0\rangle$ assigned to a reference frame $O_{0}$ to the reference frame $O_{p}$ if $\vec{v}=$ const.

The above derivation of the expression for $a_{p}(t)$ is similar to that of [19]. It is based on [37] and it is reproduced here for the convenience of readers. This is a shortened and slightly changed, simplified version of the considerations presented in [16] and mainly in [17] and more explanations and more details can be found therein and in $[39,40]$, where this formula was derived using the quantum field theory theory approach.

Inserting $\omega(\mu) \equiv \omega_{B W}(\mu)$ into (10) and into (38) one can find decay curves (survival probabilities) $\mathcal{P}_{0}(t)$ and $\mathcal{P}_{p}(t)$. Results of numerical calculations are presented in Fig (5), where calculations were performed for $\mu_{0}=0, E_{0} / \Gamma_{0} \equiv m_{0} / \Gamma_{0}=1000$ and $c p / \Gamma_{0} \equiv p / \Gamma_{0}=1000$. Values of these parameters correspond to $\gamma=\sqrt{2}$, which is very close to $\gamma$ from the experiment performed by the GSI team $[14,15]$ and this is why such values of them were chosen in our considerations. According to the literature for laboratory systems a typical value of the ratio $m_{0} / \Gamma_{0}$ is $m_{0} / \Gamma_{0} \geq O\left(10^{3}-10^{6}\right.$ ) (see eg. [41]) therefore the choice $m_{0} / \Gamma_{0}=1000$ seems to be reasonable minimum. Similarly to the case of quantum unstable systems in rest one can calculate the ratio $\mathcal{P}_{p}(t) / \mathcal{P}_{c}(t / \gamma)$ in the case of moving particles. Results of numerical calculations of this ratio are presented in Figures (6) and (7), and 

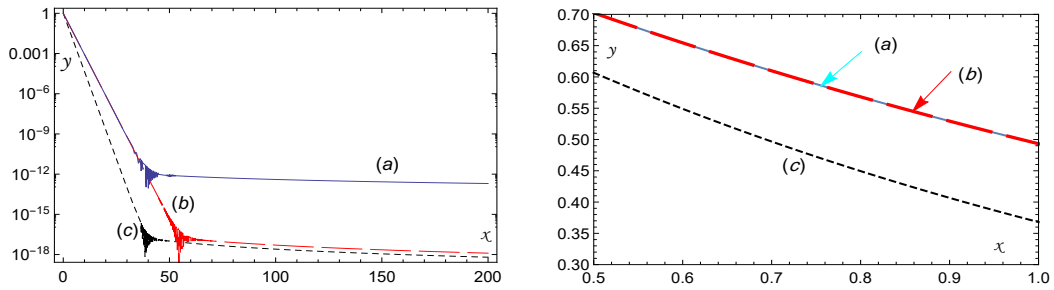

Figure 5. Decay curves obtained for $\omega_{B W}(m)$ given by Eq. (18). Axes: $x=t / \tau_{0}-$ time $t$ is measured in lifetimes $\tau_{0}, y$ - survival probabilities (The left panel: the logarithmic scales, $(a)$ the decay curve $\mathcal{P}_{p}(t),(b)$ the decay curve $\mathcal{P}_{0}(t / \gamma),(c)$ the decay curve $\mathcal{P}_{0}(t)$; The right panel: $\left.(a)-\mathcal{P}_{p}(t),(b)-\mathcal{P}_{0}(t / \gamma),(c)-\mathcal{P}_{0}(t)\right)$.

calculations were performed for $\omega(\mu)=\omega_{B W}(\mu)$ and for $\mu_{0}=0, m_{0} / \Gamma_{0}=1000, c p / \Gamma_{0} \equiv p / \Gamma_{0}=1000$ and $\gamma=\sqrt{2}$.

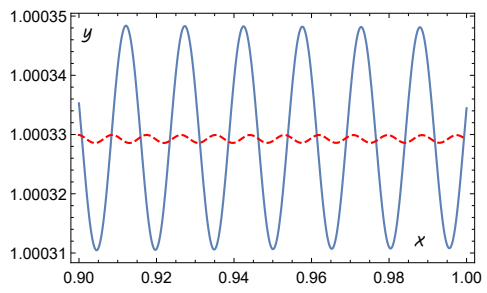

Figure 6. Axes: $x=t / \tau_{0}-$ time $t$ is measured in lifetimes $\tau_{0}, y-$ Ratio of probabilities - Solid line: $\mathcal{P}_{p}(t) / \mathcal{P}_{c}(t / \gamma)$; Dashed line $\mathcal{P}_{0}(t / \gamma) / \mathcal{P}_{c}(t / \gamma)$.
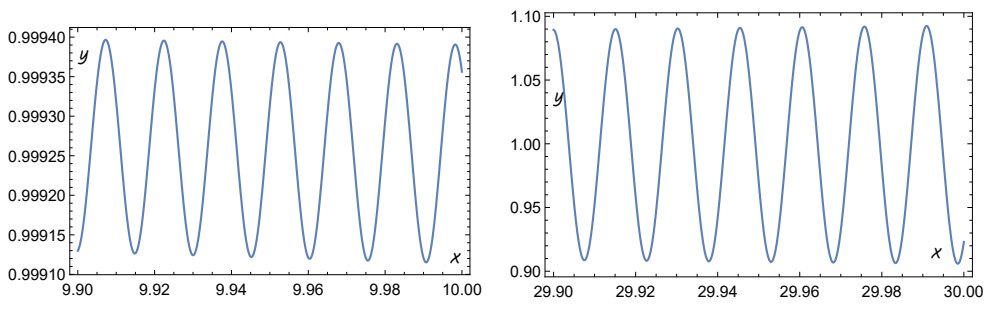

Figure 7. Axes: $x=t / \tau_{0}-$ time $t$ is measured in lifetimes $\tau_{0}, y$ - Ratio of probabilities: $\mathcal{P}_{p}(t) / \mathcal{P}_{c}(t / \gamma)$.

\section{Summary}

- The mass of the system in the unstable state $|\phi\rangle$ is not defined: It can not take the exact value. Unstable system can be characterized by the mass distribution $\omega(\mu)$, the average mass $<m>=$ $\int_{\mu_{0}}^{\infty} \mu \omega(\mu) d \mu$ and by instantaneous mass (energy) $\mu_{\phi}(t)$ but not by the mass. 
- There is no any time interval in which the survival probability (decay) law could be a decreasing function of time of the purely exponential form: Even in the case of the Breit-Wigner model in so-called "exponential regime" the decay curves are oscillatory modulated with smaller or large amplitude of oscillations depending on the parameters of the model.

- At any time interval the instantaneous mass $\mu_{\phi}(t)$ and instantaneous decay rate $\gamma_{\phi}(t)$ can not be constant in time.

- In the case of moving relativistic quantum unstable system moving with constant momentum $\vec{p}$, when unstable systems are modeled by the Brei-Wigner mass distribution $\omega(\mu)$, only at times of the order of lifetime $\tau_{\phi}$ it can be $\mathcal{P}_{p}(t) \simeq \mathcal{P}_{0}(t / \gamma)$ to a better or worse approximation. At times longer than a few lifetimes the decay process of moving particles observed by an observer in his rest system is much slower that it follows from the classical physics relation $\mathcal{P}_{p}(t) \stackrel{?}{=} \exp \left[-\frac{t}{\gamma} \Gamma_{0}\right]$.

- In the case of moving relativistic quantum unstable system moving with constant momentum $\vec{p}$ decay curves are also oscillatory modulated but the amplitude of these oscillations is higher than in the case of unstable systems in rest.

- There is a need to test the decay law of moving relativistic unstable system for times much longer than the life time

\section{Acknowledgments:}

The work was supported by the Polish NCN grant No DEC-2013/09/B/ST2/03455.

\section{References}

[1] E. Ruthheford, Philosophical Magazine, XLIX, 1, 161, (1900).

[2] E. Rutherford, F. Soddy, Philosophical Magazine, IV, 370 - 96 and 569 - 85 (1902).

[3] E. Rutherford, Radioactive substances and their radiations, Cambridge Unversity Press 1913.

[4] V. F. Weisskopf, E. T. Wigner, Z. Phys., 63, 54, (1930); 65, 18, (1930).

[5] L. A. Khalfin, Zh. Eksp. Teor. Fiz., 33, p.p. 1371 - 1382, (1957) [in Russian]; [Sov. Phys. JETP 6, (1958), 1053].

[6] L. Fonda, G. C. Ghirardii and A. Rimini, Rep. on Prog. in Phys., 41, p.p. 587 - 631, (1978).

[7] E. B. Norman, et al., Phys. Rev. Lett., 60, 2246 (1988).

[8] C. Rothe, S. I. Hintschich and A. P. Monkman, Phys. Rev. Lett., 96, 163601 (2006).

[9] B. Misra, E. C. G. Sudarshan, Journal of Mathematical Physics, 18, 745, (1977).

[10] M. C. Fischer, et al., Phys. Rev. Lett., 87, 040402, (2001).

[11] W. M. Itano, et al., Phys. Rev., A 41, 2295, (1990).

[12] Y. S. Patil, et al., Phys. Rev. Lett., 115, 140402 (2015).

[13] M. Peshkin, A. Volya and V. Zelevinsky, Europhysics Letters, 107, 40001, (2014).

[14] Yu. A. Litvinov, et al., Phys. Lett., B 664, 162, (2008).

[15] P. Kienle, et al., Phys. Lett., B 726, 638, (2013).

[16] E. V. Stefanovitch, International Journal of Theoretical Physics, 35, p.p. 2539 - 2554, (1996).

[17] M. Shirokov, International Journal of Theoretical Physics, 43, p.p. 1541 - 1553, (2004).

[18] P. Exner, Phys. Rev., D 28, p.p. 2621 - 2627, (1983).

[19] K. Urbanowski, Physics Letters, B 737, 346 - 351, (2014).

[20] J. Bailye, et al, Nature, 268 (5618), p.p. 301 - 305, (1977).

[21] David H. Frisch, James H. Smith, American Journal of Physics, 31, p.p. 342 - 355, (1963). 
[22] N. S. Krylov, V. A. Fock, Zh. Eksp. Teor. Fiz., 17, p.p. 93 - 107, (1947) [in Russian]. V. A. Fock, Fundamentals of Quantum mechanics, Mir Publishers, Moscow 1978.

[23] N. G. Kelkar, M. Nowakowski, J. Phys. A: Math. Theor., 43, 385308 (2010), 9 pp.

[24] J. Martorell, J. G. Muga, and D. W.L. Sprung, Lect. Notes Phys., 789, 239 - 275, (2009) (Springer-Verlag, Berlin, Heidelberg 2009).

[25] E. Torrontegui, J. G. Muga, J. Martorell, and D. W. L. Sprung, Advances in Quantum Chemistry, 60, $485-535,(2010)$.

[26] Gaston Garcia-Calderon, Roberto Romo and Jorge Villavicencio, Physical Review B 76, 035340 (2007), 9 pp.

[27] F. Giraldi, Eur. Phys. J. D., 69: 5, (2015), 8 pp.

[28] F. Giacosa, Found. of Phys., 42, 1262 (2012).

[29] M. L. Goldberger, K. M. Watson, Collision theory, Wiley 1964.

[30] R. E. A. C. Paley, N. Wiener, Fourier transforms in the complex domain, American Mathematical Society, New York, 1934.

[31] K. Urbanowski, Phys. Rev., A 50, 2847 - 2853, (1994).

[32] K. Urbanowski, Eur. Phys. J., C 58, 151, (2008).

[33] K. Urbanowski, Cent. Eur. J. Phys., 7, 696 - 703, (2009).

[34] K. M. Sluis, E. A. Gislason, Phys. Rev. A 43, (1991), 4581.

[35] NIST Handbook of Mathematical Functions, Natl. Inst.. Stand. and Technology, Eds.: F. W. J. Olver, D. W. Lozier, R. F. Boisvert and Ch. W. Clark,(Cambridge, 2010).

[36] Handbook of Mathematical Functions With Formulas, Graphs, and Mathematical Tables, Eds.: Milton Abramowitz and Irene A. Stegun, New York: Dover Publications, Inc. (1964).

[37] W. M. Gibson, B. R. Polard, Symmetry principles in elementary particle physics, Cambridge, 1976.

[38] L. A. Khalfin, Quantum theory of unstable particles and relativity, PDMI PREPRINT-6/1997 (St. Petersburg Department of Steklov Mathematical Institute, St. Petersburg, Russia, 1997).

[39] E. V. Stefanovich, Relativistic quantum dynamics, arXiv: 0504062 [physics]. E. V. Stefanovich, Violations of Eistein's time dilation formula in particle decays, arXiv: 0603043 [physics]. B. T. Shields, et al, Phys. Rev., A 82, 052116 (2010).

[40] M. I. Shirokov, Concepts of Physics, 3, 193 - 205 (2006); arXiv: quant-ph/0508087v.2.; Physics of Particles and Nuclei Letters, 6, $14-17,(2009)$.

[41] L. M. Krauss, J. Dent, Phys. Rev. Lett., 100, 171301 (2008), 4 pp. 